\title{
Phosphoglucose isomerase enhances colorectal cancer metastasis
}

\author{
SOICHI TSUTSUMI, TAKAHARU FUKASAWA, HAYATO YAMAUCHI, TOSHIHIDE KATO, \\ WAKAKO KIGURE, HIROKI MORITA, TAKAYUKI ASAO and HIROYUKI KUWANO
}

Department of General Surgical Science (Surgery I), Gunma University Graduate School of Medicine, 3-39-22 Showa-machi, Maebashi, Gunma 371-8511, Japan

Received May 13, 2009; Accepted July 30, 2009

DOI: 10.3892/ijo_00000427

\begin{abstract}
Phosphoglucose isomerase (PGI) is a ubiquitous cytosolic enzyme that plays a key role in glycolysis. PGI is also a multifunctional protein that acts in the extracellular milieu as a potent mitogen/cytokine. Increased expression of PGI and its receptor has been found in a wide spectrum of malignancies and is associated with cancer progression and metastasis. In this study, the role of PGI in the growth and metastasis of colon cancer cells was determined. To elucidate the functional role of PGI in colorectal cancer, we stably transfected PGI cDNA into human colon cancer cells. We used an orthotopic mouse tumor model to assess whether overexpression of PGI enhances liver metastasis. Overexpression of PGI stimulated the in vitro invasion of DLD-1 cells. In vivo, after orthotopic implantation into the cecum of nude mice, parental and empty vector-transfected DLD-1 cells produced small tumors without liver metastasis, whereas PGI-overexpressing DLD-1 cells produced large tumors and liver metastases. In conclusion, overexpression of PGI significantly contributes to the aggressive phenotype of human colon cancer and, thus, may provide a novel therapeutic target.
\end{abstract}

\section{Introduction}

Colorectal cancer (CRC) is the third most commonly diagnosed cancer and the third leading cause of cancer-related death in both men and women in the US, with an estimated 49,960 deaths in 2008 (1). Approximately $30 \%$ of all patients with CRC have metastatic disease at diagnosis, and $50 \%$ of early stage patients eventually develop metastatic or advanced disease (2). The introduction of new chemotherapeutic regimens, including FOLFOX + bevacizumab and FOLFIRI + bevacizumab, has increased the median survival of these patients (3). However, the results of chemotherapy remain

Correspondence to: Dr Soichi Tsutsumi, Department of General Surgical Science (Surgery I), Gunma University Graduate School of Medicine, 3-39-22 Showa-machi, Maebashi, Gunma 371-8511, Japan

E-mail: chuchumi@showa.gunma-u.ac.jp

Key words: phosphoglucose isomerase, colorectal cancer, metastasis inferior to those of curative hepatic resection, which results in 5 -year survival rates of 40 to $50 \%$ in selected patients $(4,5)$. More effective modalities of treatment for metastatic CRC are needed.

Cancerous tissue exhibits a significantly higher glucose consumption than normal tissue. Moreover, a higher glucose uptake correlates with higher tumor aggressiveness and progressively poorer prognosis. Cancer cells maintain high aerobic glycolytic rates and produce high levels of lactate and pyruvate. Aerobic glycolysis in tumors can be regulated at the level of glycolytic enzymes or glucose transporters in tumor cells. Phosphoglucose isomerase (PGI; EC 5.3.1.9) is a cytosolic enzyme that plays a key role in both glycolysis and gluconeogenesis pathways (6). Molecular cloning and sequencing have identified PGI with the autocrine motility factor (AMF). AMF is a malignant factor that enhances the invasive and metastatic abilities of tumor cells $(7,8)$. AMF is an orphan $\mathrm{CXXC}$ chemokine, and its cognate receptor is a unique seven-transmembrane glycoprotein receptor (autocrine motility factor receptor, AMFR). Overexpression of AMF/ PGI and AMFR has been found in a number of different malignancies and is associated with cancer progression and metastasis $(7,9,10)$. In this study, we examined whether PGI contributes to the distant metastasis of CRC.

\section{Materials and methods}

Materials. The following antibodies were used. Anti-ß-actin was from Santa Cruz Biotechnology, Inc. (Santa Cruz, CA), and anti-AMF and anti-AMFR were described previously (7). We purified anti-AMF IgG and preimmune IgG from serum using ImmunoPure $(\mathrm{G}) \mathrm{IgG}$ according to the manufacturer's instructions (Pierce, Rockford, IL).

Cell culture and transfection. DLD-1 human colon carcinomas were purchased from the Health Science Research Resources Bank (Osaka, Japan) and maintained in RPMI-1640 supplemented with $10 \%$ fetal bovine serum (FBS), essential and nonessential amino acids, and penicillin/streptomycin. The cells were maintained in a humidified chamber with $95 \%$ air and $5 \% \mathrm{CO}_{2}$ at $37^{\circ} \mathrm{C}$.

The full-length human PGI cDNA was generated by PCR amplification (11). The PCR product was ligated into a mammalian expression vector pcDNA3.1 zeo (Invitrogen, Carlsbad, CA). According to the manufacturer's instructions, Lipofectamine 2000 (Life Technologies, Inc., Gaithersburg, 
MD) was used to transfect parental DLD-1 cells with PGI cDNA. Isolation of single clones of the stable transfectants was accomplished by adding $750 \mu \mathrm{g} / \mathrm{ml}$ of Zeocin (Invitrogen) to the culture medium. The DLD-1 cell line, stably transfected with pcDNA3.1 zeo or pcDNA3.1 zeo-PGI, was designated DLD-zeo or DLD-PGI, respectively. All experiments were repeated at least three times, and results were confirmed by both clonal cell lines and the pooled cell population.

Western blot analysis. The cells were lysed in a lysis buffer [50 mmol/1 HEPES (pH 7.9), 0.1\% NP40, 10\% glycerol, $1 \mathrm{mmol} / \mathrm{l} \mathrm{DTT}, 1 \%$ sodium deoxycholate, $250 \mathrm{mmol} / \mathrm{l} \mathrm{NaCl}$, $5 \mathrm{mmol} / \mathrm{l} \mathrm{EDTA}, 1 \mathrm{mmol} / \mathrm{l}$ phenylmethylsulfonyl fluoride and $0.1 \mathrm{mg} / \mathrm{ml}$ leupeptin] at $4^{\circ} \mathrm{C}$. Cell lysates containing equal amounts of protein were separated by SDS-PAGE and transferred to a polyvinylidene fluoride membrane (MSI, Westborough, MA). The membranes were blocked with 5\% nonfat dry milk in Tris-buffered saline with $0.05 \%$ Tween-20 (TBS-T), incubated with the primary antibody for $2 \mathrm{~h}$, washed 3 times within $15 \mathrm{~min}$ in TBS-T, incubated with the secondary horseradish peroxidase-conjugated antibody (Zymed, San Francisco, CA) for $1 \mathrm{~h}$, and finally washed three times. The horseradish peroxidase activity was detected by incubation of the membrane with an enhanced chemiluminescence reagent (Amersham, Arlington Heights, IL). A Kodak imaging system was used to determine the density of the bands.

Cell proliferation assays. Cell proliferation assays were conducted by seeding cells at a density of $1 \times 10^{5}$ cells/well in 6 -well plates. Cells were fed RPMI-1640 with $10 \%$ FBS every other day and counted daily.

Invasion assay. The invasive activity of cells was assayed in transwell cell culture chambers (Corning Costar Co., Cambridge, MA). Polycarbonate filters with an $8-\mu \mathrm{m}$ pore size were coated with Matrigel $(1 \mathrm{mg} / \mathrm{ml}$ ) (Collaborative Biomedical Products, Bedford, MA) to form a matrix barrier. Cells were resuspended to a concentration of $1 \times 10^{6} / \mathrm{ml}$ in RPMI-1640 with $0.5 \%$ FBS. The cell suspension (100 $\mu 1)$ was added to the upper compartment of the chamber and incubated with RPMI-1640 with $10 \%$ FBS in the lower compartment for $24 \mathrm{~h}$ at $37^{\circ} \mathrm{C}$. The filters were fixed with $4 \%$ paraformaldehyde and stained with hematoxylin. The cells on the upper surface of the filters were removed by wiping with cotton swabs. The cells that had invaded through the Matrigel and the filter to the lower surface were counted. Each assay was conducted in triplicate. In some experiments, anti-AMF $\operatorname{IgG}(50 \mu \mathrm{g} / \mathrm{ml})$ or preimmune $\operatorname{IgG}(50 \mu \mathrm{g} / \mathrm{ml})$ was incubated in the medium.

Glucose consumption, lactate production, and $\mathrm{pH}$ value in vitro. Parental and variant cell lines were grown to near confluence in RPMI-1640 culture medium buffered with $25 \mathrm{mM} \mathrm{NaHCO}_{3}$ and supplemented with $10 \%$ FBS. Cultures were then rinsed with PBS and provided with a fresh culture medium. The glucose and lactate levels and $\mathrm{pH}$ values were subsequently assayed in the conditioned medium by a blood gas analyzer (Stat Profile M; Nova Biomedical, Waltham, MA) every $2 \mathrm{~h}$.

\section{Orthotopic tumor model}

Animals and orthotopic implantation of tumor cells. Female athymic nude mice were housed under specific pathogen-free conditions and used at 6 weeks of age. Subcutaneous xenografts were established by injection of $1 \times 10^{6}$ tumor cells in both flanks of 2-3 animals per cell line, and the growth was monitored regularly. Tumors were selected for orthotopic implantation at an average size of $1 \mathrm{~cm}^{3}$, and tissue was cut into $2 \times 2 \times 2 \mathrm{~mm}$ pieces. Superficial regions of the tumors were used for implantation, and all sampled tumors were examined histologically to confirm that areas of the tumor corresponding to the sampled regions contained viable tissue. The cecum was exteriorized through a small midline laparotomy, and a piece of tumor tissue was sutured to the cecal surface with a single Maxon ${ }^{\mathrm{TM}}$ 6-0 suture, leaving the tumor tissue buried in the cecal wall. After implantation, the abdominal wall was closed in two layers. The volume (V) of the xenograft was calculated by the following formula: $\mathrm{V}=$ _/6(a x b x c), where a, b, and c represent the length, width, and height of the mass, respectively. The mean values and the SDs of the tumor volumes were calculated. Ten mice were inoculated with each type of cell in two separate experiments.

Histopathologic and immunohistochemical study. The tumors were fixed in $10 \%$ phosphate-buffered formalin, and 4- $\mu \mathrm{m}$ paraffin-embedded sections were prepared. Slides were stained with $\mathrm{H} \& \mathrm{E}$ according to standard laboratory protocols. We completed an immunohistochemical study using a Histofine SAB-PO Kit according to the manufacturer's instructions (Nichirei Co., Tokyo, Japan).

Clinical samples. Colorectal cancer tissues were obtained from 10 patients undergoing initial surgical resection without any prior therapy. Formalin-fixed, paraffin-embedded tissue sections were subjected to immunostaining using anti-AMF $\mathrm{IgG}$ as described above.

Statistical analysis. A Student's t-test was used in the statistical analyses. $\mathrm{P}<0.05$ was considered significant.

\section{Results}

Constitutive overexpression of PGI in DLD-1 transfectants. After transfection, three stably transfected cell clones (DLDPGI1, DLD-PGI2, and DLD-PGI3) of human PGI cDNA exhibiting high-level expression of AMF in DLD-1 cells were selected and established. The PGI ratios of each clone were 3 to 4 compared with the empty vector-transfected control (Fig. 1). No differences in expression of AMFR were observed among the PGI transfectants and vector-only transfected cells. We conducted tests to determine whether overexpression of PGI in DLD-1 human colon cancer cells affects their in vitro growth properties. No statistical significance in the growth rate among the cell variants was detected (data not shown). PGI increased the invasive ability of DLD-1 cells by 1.5 - to 2 -fold relative to the parental DLD-1 and DLD-zeo (Fig. 1C).

In vitro metabolism of parental and PGI-overexpressing cells. The glucose metabolism in the PGI-overexpressing and 
A

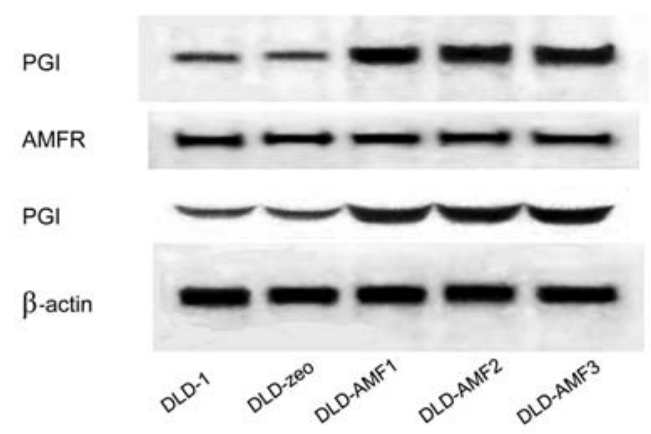

B

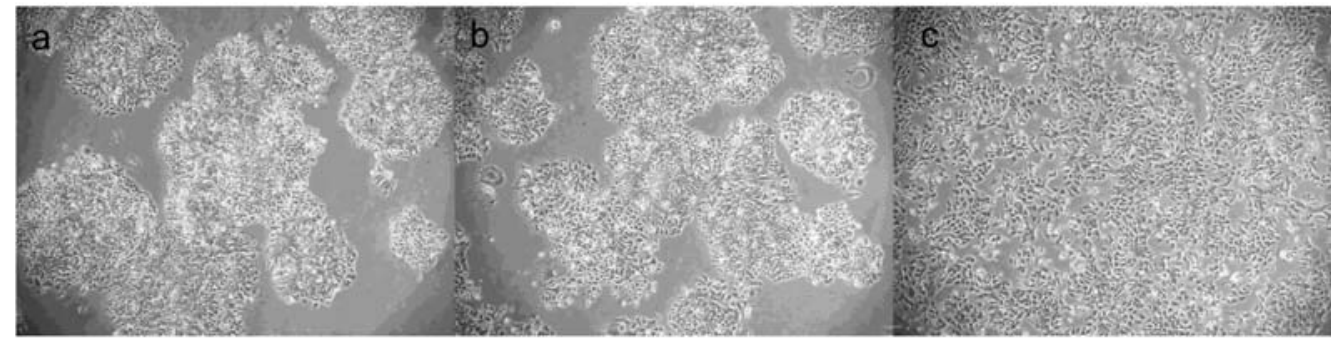

DLD-1 Parental cell

DLD-zeo

DLD-PGI1

C

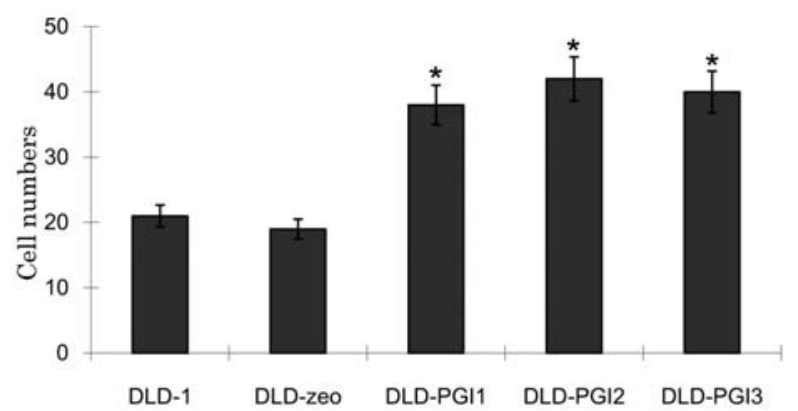

Figure 1. (A) PGI expression in colon cancer cells transfected with PGI expression vectors. The parental DLD-1 cells were stably transfected with empty vector (DLD-zeo), and AMF expression vector (DLD-PGI1, DLD-PGI2, and DLD-PGI3, three individual clones), and cell lysates from these cells were subjected to Western blot analysis with anti-PGI antibody (top row) or anti-AMFR antibody (second row). The secretion of AMF was analyzed by Western blotting of $50 \mu \mathrm{g}$ of protein from conditioned medium (third row). The fourth row was probed with anti- $\beta$-actin antibody as a control. (B) Morphological changes induced by PGI overexpression. Morphological alterations after PGI overexpression were observed in the transfected cell lines. Parental DLD-1 (a) and DLD-zeo (b) cells were more adherent to each other and formed compact clusters, whereas the PGI-transfected cells (c) continued to exhibit a dispersed morphology. (C) Invasive ability of PGI-transfected DLD-1 cells. A single-cell suspension ( $200 \mu 1)\left(1 \times 10^{6}\right.$ cells $\left./ \mathrm{ml}\right)$ of cells was placed in the upper wells of individual transwell inserts containing $8-\mu \mathrm{m}$ pore polycarbonate membranes precoated with Matrigel. Cells were allowed to invade for $24 \mathrm{~h}$ at $37^{\circ} \mathrm{C}$, and then they were fixed and stained with hematoxylin. Cells on the upper surface were removed with a cotton swab, and the cells that migrated to the lower side of the membrane were mounted on a microscope slide and counted under a light microscope at x 200 magnification. In vitro invasive ability was significantly greater in PGI-transfected cells when compared with parental and empty vector-transfected DLD-1 cells at 24 h. ${ }^{*}$ Significant difference when compared with the control $(\mathrm{P}<0.005)$. Each value represents a mean of three readings. Error bars represent $95 \%$ confidence intervals. ${ }^{*} \mathrm{P}<0.001$.

Table I. Effect of PGI overexpression on colonic tumors in nude mice.

\begin{tabular}{lcccc}
\hline \multirow{2}{*}{ Cell line } & \multicolumn{2}{c}{ Cecal tumors } & & $\begin{array}{c}\text { Liver } \\
\text { metastasis }\end{array}$ \\
\cline { 2 - 3 } \cline { 5 - 5 } & Incidence & Volume $\left(\mathrm{mm}^{3}\right)$ & & Incidence \\
\hline DLD-1 & $10 / 10$ & $39.3 \pm 3.5$ & & $0 / 10$ \\
DLD-zeo & $10 / 10$ & $36.7 \pm 1.9$ & & $0 / 10$ \\
DLD-PGI1 & $10 / 10$ & $93.1 \pm 13.4^{\mathrm{a}}$ & & $6 / 10$ \\
DLD-PGI2 & $10 / 10$ & $92.3 \pm 16.5^{\mathrm{a}}$ & & $5 / 10$ \\
DLD-PGI3 & $10 / 10$ & $90.9 \pm 19.3^{\mathrm{a}}$ & & $6 / 10$ \\
\hline
\end{tabular}

${ }^{\mathrm{a}} \mathrm{P}<0.01$ versus PGI-zeo. control vector clones was compared by measuring the glucose and lactate levels and $\mathrm{pH}$ values in an in vitro time course assay. Both glucose consumption and lactate production were significantly lower in the parental and vector-only cells than in the PGI-overexpressing cells (Fig. 2A and B). The PGI-overexpressing cells acidified the medium more strongly than did the parental and vector-only cells (Fig. 2C).

Effect of PGI expression on the in vivo growth of DLD-1 cells. To determine the metastatic potential of parental DLD-1, DLD-zeo, and DLD-PGI cells $\left(2 \times 10^{6}\right.$ cells/mouse $)$, these cells were orthotopically implanted into the cecum of nude mice ( $\mathrm{n}=10$ for each variant). Orthotopically implanted control cells (parental DLD-1 and DLD-zeo) formed small tumors, and PGI-overexpressing cells produced larger tumors (Fig. 3Aa and b). The metastatic potential of the cells was 
A

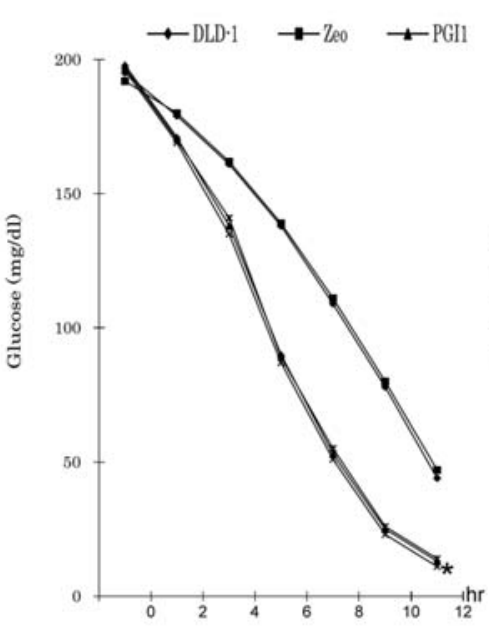

B

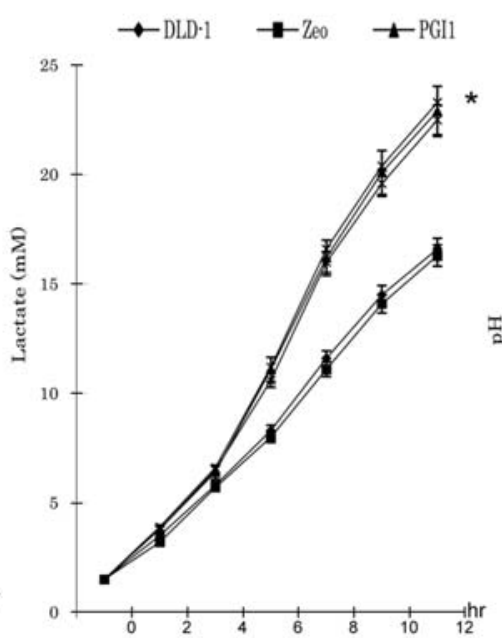

C

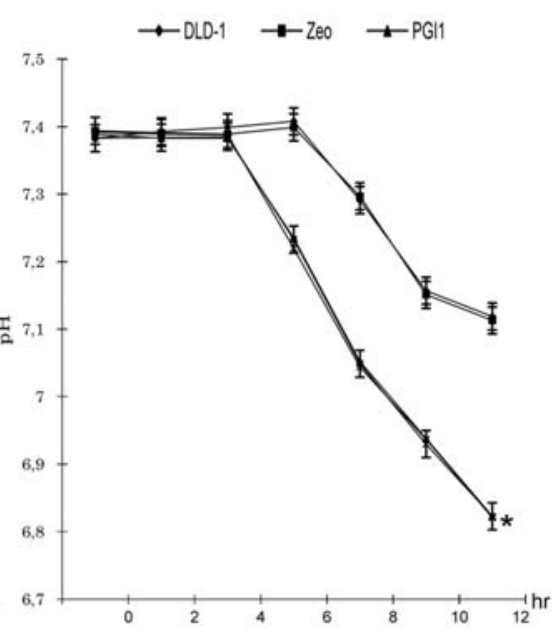

Figure 2. Glucose consumption (A), lactate production (B), and medium acidification (C) of the derived DLD-1, DLD-zeo and DLD-PGI cells in vitro. Cells were grown as described in Materials and methods. The mean \pm SD (bars) for each time point is shown ( $n=3)$. * Significant difference when compared with the control $(\mathrm{P}<0.0001$ at $12 \mathrm{~h})$.

A

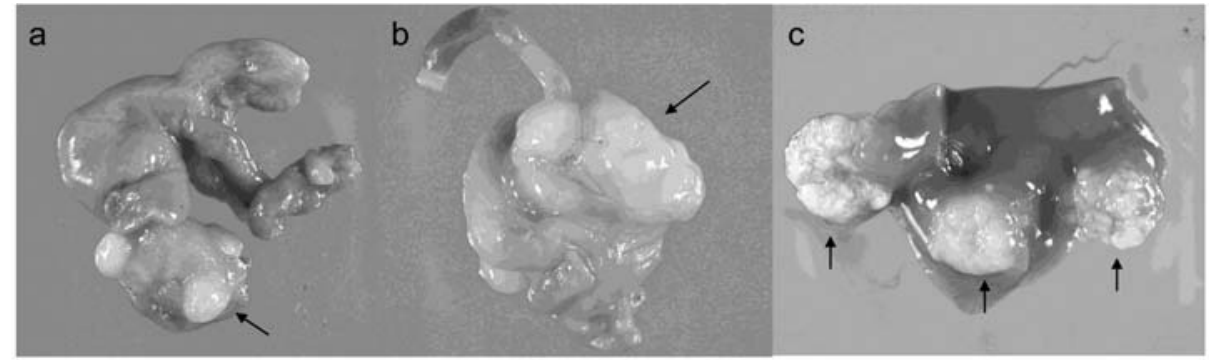

B

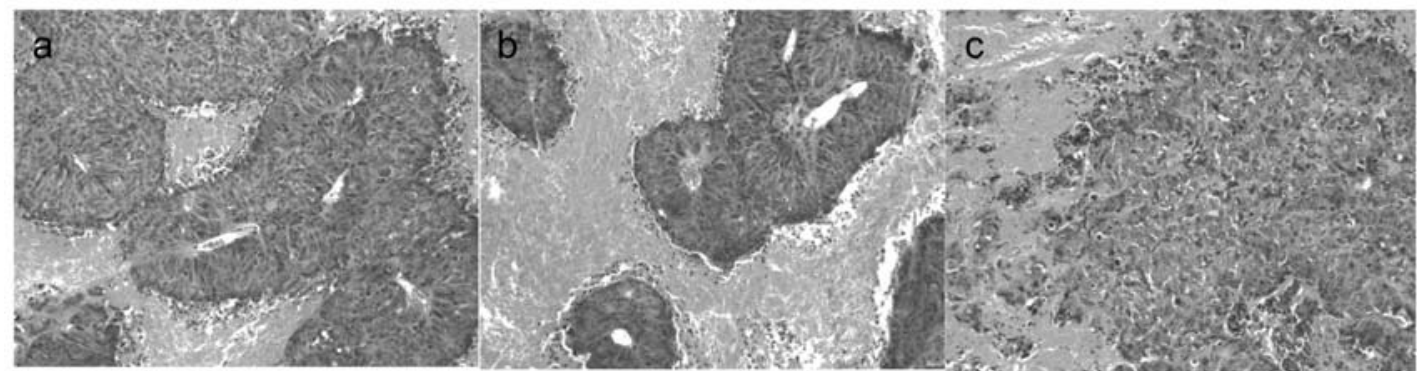

Figure 3. (A) PGI enhanced growth and metastasis in vivo. To evaluate the effect of PGI signaling on local and distant metastatic tumor growth, a more clinically relevant orthotopic tumor model was selected. Empty vector-transfected DLD-1 cells formed small tumors (a, arrow), whereas the PGI-transfected cells formed more aggressive tumors (b, arrow). PGI-transfected DLD-1 cells produced spontaneous liver metastases (c, arrow), whereas the parental and empty vector-transfected DLD-1 cells were nonmetastatic in a reproducible manner. (B) Histologic appearance of PGI-overexpressing tumors in nude mice. Tumors were generated by orthotopical inoculation of PGI-transfected DLD-1 cells as described in Materials and methods. Parental (a) and empty vector-transfected (b) DLD-1 tumors did not invade surrounding tissues and showed localized tumor growth, whereas PGI-transfected cells focally invaded the surrounding colonic tissue (c).

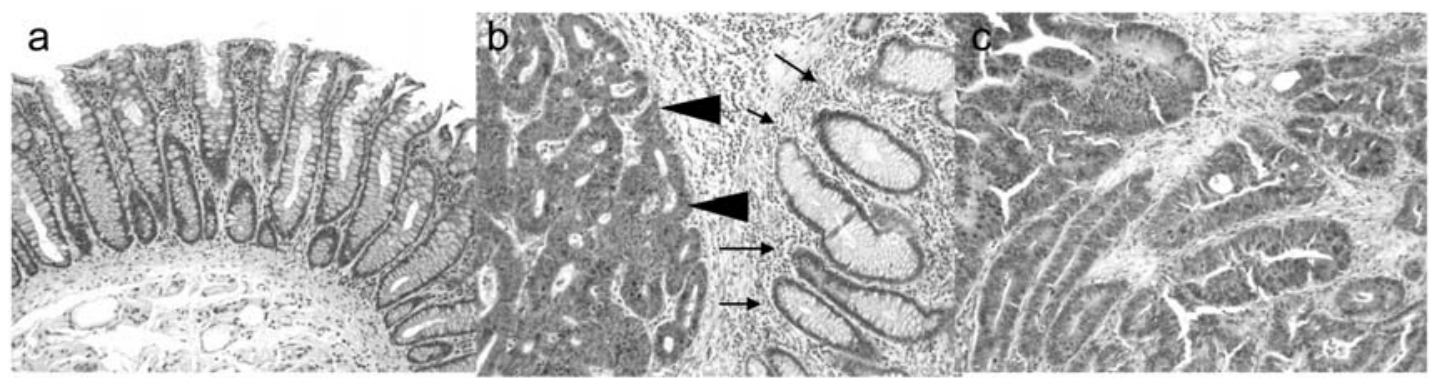

Figure 4. Expression of PGI in human colon cancers. Representative example of PGI expression of human colon cancer determined by immunohistochemistry. a, Normal colonic mucosa; b, moderately differentiated adenocarcinoma (arrow); c, moderately differentiated adenocarcinoma. Strongly positive PGI expression is present in the cytoplasm of cancer cells (b and c) but not in normal colonic epithelial cells (a). 
determined 1 month after orthotopic implantation of tumor cells. DLD-PGI cells produced spontaneous liver metastases (Fig. 3Ac), whereas the parental DLD-1 and DLD-zeo cells were never metastatic. These data show that increased expression of PGI leads to the enhancement of tumor formation and metastasis of human colon cancer cells (Table I). Parental DLD-1 and DLD-zeo tumors did not invade surrounding tissues and showed localized tumor growth (Fig. 3Ba and b), whereas DLD-PGI cells focally invaded surrounding colonic tissue (Fig. 3Bc).

Overexpression of PGI in human colon cancer. Expression of the PGI protein was examined in paraffin sections from 10 colon cancer patients by immunohistochemistry. Strong cytoplasmic staining of the PGI protein was detected in 10 cancers but weakly expressed in normal colonic epithelium (Fig. 4). A representative example of PGI expression in human colon cancer was determined by immunohistochemistry. Strongly positive PGI expression was observed in the cytoplasm of cancer cells (Fig. $4 \mathrm{~b}$ and c) but not in that of normal colonic cells (Fig. 4a).

\section{Discussion}

The recent advent of new agents for the treatment of metastatic CRC has enhanced the therapeutic strategy for this disease. Oxaliplatin in the FOLFOX and FOLFIRI regimen has been shown to be effective in achieving an improved response and time to progression (3). The monoclonal antibodies bevacizumab (targeting the vascular endothelial growth factor) and cetuximab (targeting EGFR) have also shown therapeutic efficacy in CRC (4). The effort to further improve the efficacy and tolerability of treatment for metastatic CRC has led to the discovery of new agents targeting cell-signaling molecules.

Cancer metastasis is a multistep process involving complex and highly coordinated interactions between tumor cells and a constantly changing host microenvironment (12). It has also been reported that the aberrant expression of glycolytic enzymes is regulated by hypoxia, including PGI expression (13). The adaptation of cancer cells to hypoxia is regulated by hypoxia-inducible factor-1, a key transcription factor that up-regulates the glycolytic enzyme that improves cell survival and promotes the progression of cancers that rely on aerobic glycolysis (14). Likewise, the increased expression of this glycolytic enzyme supports the survival and growth of these cells under hypoxic conditions in cancers (15). The demonstration that PGI-overexpressing DLD-1 cells exhibit enhanced glucose consumption and lactate production properties in vitro highlights the possible role of PGI overexpression during cancer progression and metastasis. Targeting of growth factor receptors with monoclonal antibodies for cancer treatment has become possible with the recent introduction of humanized antibodies.
The present study highlights the role of PGI expression in a key event which controls tumor progression and metastasis. The results herein call for the development of specific antiPGI antibodies or a PGI inhibitor specifically targeting metastatic CRC. Such inhibitors are being actively investigated at the molecular level and may hold promise as effective anticancer treatments. In addition, research is required to determine whether PGI can be a therapeutic target candidate.

\section{References}

1. Jemal A, Siegel R, Ward E, Hao Y, Xu J, Murray T and Thun MJ: Cancer statistics 2008. CA Cancer J Clin 58: 71-96, 2008.

2. Hamady ZZ, Kotru A, Nishio H and Lodge JP: Current techniques and results of liver resection for colorectal liver metastases. $\mathrm{Br}$ Med Bull 70: 87-104, 2004.

3. Saltz LB, Clarke S, Díaz-Rubio E, Scheithauer W, Figer A, Wong R, Koski S, Lichinitser M, Yang TS, Rivera F, Couture F, Sirzén F and Cassidy J: Bevacizumab in combination with oxaliplatin-based chemotherapy as first-line therapy in metastatic colorectal cancer: a randomized phase III study. J Clin Oncol 26: 2013-2019, 2008

4. Kelly H and Goldberg RM: Systemic therapy for metastatic colorectal cancer: current options, current evidence. J Clin Oncol 23: 4553-4560, 2005.

5. Abdalla EK, Vauthey JN, Ellis LM, et al: Recurrence and outcomes following hepatic resection, radiofrequency ablation, and combined resection/ablation for colorectal liver metastases. Ann Surg 239: 818-825, 2004.

6. Harrison RA: The detection of hexokinase, glucosephosphate isomerase and phosphoglucomutase activities in polyacrylamide gels after electrophoresis: a novel method using immobilized glucose 6-phosphate dehydrogenase. Anal Biochem 61: 500-507, 1974.

7. Tsutsumi S, Hogan V, Nabi IR and Raz A: Overexpression of the autocrine motility factor/phosphoglucose isomerase induces transformation and survival of NIH-3T3 fibroblasts. Cancer Res 63: 242-249, 2003.

8. Tsutsumi S, Yanagawa T, Shimura T, Kuwano H and Raz A: Autocrine motility factor signaling enhances pancreatic cancer metastasis. Clin Cancer Res 10: 7775-7784, 2004.

9. Taniguchi K, Yonemura Y, Nojima N, Hirono Y, Fushida S, Fujimura T, Miwa K, Endo Y, Yamamoto $\mathrm{H}$ and Watanabe $\mathrm{H}$ : The relation between the growth patterns of gastric carcinoma and the expression of hepatocyte growth factor receptor (c-met), autocrine motility factor receptor, and urokinase-type plasminogen activator receptor. Cancer 82: 2112-2122, 1998.

10. Nakamori S, Watanabe H, Kameyama M, Imaoka S, Furukawa H, Ishikawa O, Sasaki Y, Kabuto T and Raz A: Expression of autocrine motility factor receptor in colorectal cancer as a predictor for disease recurrence. Cancer 74: 1855-1862, 1994.

11. Niinaka Y, Paku S, Haga A, Watanabe H and Raz A: Expression and secretion of neuroleukin/phosphohexose isomerase/ maturation factor as autocrine motility factor by tumor cells. Cancer Res 58: 2667-2674, 1998.

12. Subarsky P and Hill RP: The hypoxic tumour microenvironment and metastatic progression. Clin Exp Metastasis 20: 237-250, 2003.

13. Niizeki H, Kobayashi M, Horiuchi I, et al: Hypoxia enhances the expression of autocrine motility factor and the motility of human pancreatic cancer cells. Br J Cancer 86: 1914-1919, 2002.

14. Lu H, Forbes RA and Verma A: Hypoxia-inducible factor 1 activation by aerobic glycolysis implicates the Warburg effect in carcinogenesis. J Biol Chem 277: 23111-23115, 2002.

15. Malhotra R and Brosius FC III: Glucose uptake and glycolysis reduce hypoxia-induced apoptosis in cultured neonatal rat cardiac myocytes. J Biol Chem 274: 12567-12575, 1999. 